Dementia

and Geriatric

Cognitive Disorders
Dement Geriatr Cogn Disord 2020;49:38-47

DOI: 10.1159/000508392
Received: April 7, 2020

Accepted: May 1, 2020

Published online: July 1, 2020

\title{
The Power of EEG to Predict Conversion from Mild Cognitive Impairment and Subjective Cognitive Decline to Dementia
}

\author{
Knut Engedala, b Maria Lage Barca ${ }^{a, b}$ Peter Høgh ${ }^{c, d} \quad$ Birgitte Bo Andersen ${ }^{\mathrm{a}}$ \\ Nanna Winther Dombernowsky ${ }^{\mathrm{e}}$ Mala Naik ${ }^{\mathrm{f} g}$ Thorkell Eli Gudmundsson ${ }^{\mathrm{h}}$ \\ Anne-Rita Øksengaard ${ }^{\mathrm{i}}$ Lars-Olof Wahlund ${ }^{j} \quad$ Jon Snaedal ${ }^{\mathrm{h}}$ \\ ${ }^{a}$ Norwegian Advisory Unit for Aging and Health, Vestfold Health Trust, Tønsberg, Norway; ${ }^{b}$ Department of Geriatric \\ Medicine, Oslo University Hospital, Oslo, Norway; ${ }^{C}$ Department of Neurology, Regional Dementia Research Center, \\ Zealand University Hospital, Roskilde, Denmark; ${ }^{\mathrm{d}}$ Department of Clinical Medicine, University of Copenhagen, \\ Copenhagen, Denmark; ${ }^{e}$ Department of Neurology, Danish Dementia Research Center, Copenhagen University \\ Hospital, Rigshospitalet, Copenhagen, Denmark; ${ }^{\mathrm{f}}$ Department of Geriatric Medicine, Haraldsplass Deaconess \\ Hospital, Bergen, Norway; ${ }^{9}$ Department of Clinical Science, University of Bergen, Bergen, Norway; ${ }^{\text {h Department }}$ \\ of Geriatric Medicine, Landspitali University Hospital, Reykjavik, Iceland; 'Norwegian National Health Association,

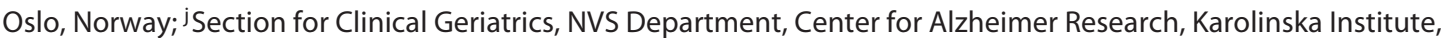 \\ Stockholm, Sweden
}

\section{Keywords}

Mild cognitive impairment · Subjective cognitive decline ·

Dementia $\cdot$ EEG

\begin{abstract}
Introduction: The aim of this study was to examine if quantitative electroencephalography (qEEG) using the statistical pattern recognition (SPR) method could predict conversion to dementia in patients with subjective cognitive decline (SCD) and mild cognitive impairment (MCI). Methods: From 5 Nordic memory clinics, we included 47 SCD patients, 99 $\mathrm{MCl}$ patients, and 67 healthy controls. EEGs analyzed with the SPR method together with clinical data recorded at baseline were evaluated. The patients were followed up for a mean of 62.5 (SD 17.6) months and reexamined. Results: Of 200 participants with valid clinical information, 70 had converted to dementia, and 52 had developed Alzheimer's disease. Receiver-operating characteristic analysis of the EEG results as defined by a dementia index (DI) ranging from 0 to
\end{abstract}

100 revealed that the area under the curve was $0.78(95 \% \mathrm{Cl}$ $0.70-0.85)$, corresponding to a sensitivity of $71 \%$, specificity of $69 \%$, and accuracy of $69 \%$. A logistic regression analysis showed that by adding results of a cognitive test at baseline to the EEG DI, accuracy could improve. Conclusion: We conclude that applying qEEG using the automated SPR method can be helpful in identifying patients with $S C D$ and $M C l$ that have a high risk of converting to dementia over a 5-year period. As the discriminant power of the method is of moderate degree, it should be used in addition to routine diagnostic methods.

(c) 2020 S. Karger AG, Basel

\section{Introduction}

The World Health Organization has declared dementia to be one of the biggest health challenges in the future to come because neurodegenerative disorders are the most prominent underlying conditions in which no modifying

\section{KARGER}

(c) 2020 S. Karger AG, Basel

karger@karger.com

www.karger.com/dem
Knut Engedal

Norwegian Advisory Unit for Aging and Health

Vestfold Health Trust, PO 2136

NO-3103 Tønsberg (Norway)

knut.engedal@aldringoghelse.no 
treatment exists [1]. Due to the increasing number of older people worldwide, the prevalence of dementia will increase from about 45 million people today to $>130$ million during the next 30 years $[2,3]$. Alzheimer disease $(\mathrm{AD})$ is the most frequent cause of dementia followed by vascular dementia (VaD), dementia with Lewy bodies (DLB), and other forms of neurodegenerative disorders. Typically, $\mathrm{AD}$ develops slowly, and brain pathology can be found decades before clinical symptoms are seen in patients [4]. If or when we find modifying treatments, such treatments should be initiated before the disease has progressed to a clinical state with severe cognitive decline and impaired function in activities of daily living. Therefore, it is of importance to find valid biomarkers that could identify people that have a high risk of developing dementia and, preferably, the underlying cause of dementia.

Prior to the stage of dementia, 2 stages of impaired cognition are frequently defined: subjective cognitive decline (SCD), a stage of subjectively experienced impaired cognition, which is not supported by an informant or by neuropsychological testing, and mild cognitive impairment (MCI), which is supported by an informant and in-depth testing. In both stages, the individuals are able to perform personal as well as instrumental activities of daily life, but they are at high risk of developing dementia [5-8]. Using standardized criteria, these individuals can be identified [9-14]. Previous studies have shown that $20-50 \%$ of people with MCI will convert to dementia $[6-8,15]$. Older age, poorer cognition, APOE $\varepsilon 4$ allele carrier status, and hypertension are found to increase the conversion rate to dementia [15]. The use of various MRI and PET methods and cerebrospinal fluid (CSF) examination, measuring concentrations of amyloid- $\beta$, phosphorylated and total tau proteins, as well as other proteins, including inflammatory markers, have shown to be helpful in predicting patients with MCI and SCD who will convert to dementia, especially to AD [16-22]. A recent Cochrane review, however, does not support the use of $\beta$-amyloid PET as biomarker for $\mathrm{AD}$, but studies examining the combination of cognitive tests with CSF proteins, MRI, and FDG-PET have shown excellent results in identifying people with MCI that would convert to dementia [2325]. However, the use of biomarkers are time and personnel consuming, costly, and some of them are invasive, and, consequently, not ideal for use in daily clinical practice, except in subspecialized settings. Therefore, biomarkers are generally not used in primary care settings and not at all in countries with poor economy, and some will not be applied where doctors are reluctant to use invasive methods such as tapping cerebrospinal fluid. Electroencephalography (EEG), on the other hand, is a low-cost, noninvasive, simple examination that can be used in most clinics and even in primary care settings, especially if an automatic reading method is applied. In the last decade, studies were published which examined the power of EEG to detect those persons with MCI that will convert to dementia [26-30]. Further, our Nordic group has examined the validity of automated quantitative EEG (qEEG) using the statistical pattern recognition (SPR) method, and various results have demonstrated how this method can be used to separate patients with dementia from healthy controls (HC) [31-37]. Especially, qEEG seems to be helpful in diagnosing DLB $[29,35,38,39]$. The SPR method has, however, never been applied to examine if the method can be used to separate patients with SCD and MCI that will later convert to dementia or not. Thus, the aim of this study was to examine if qEEG using the SPR method could predict conversion to dementia in patients with SCD and MCI.

\section{Material and Methods}

This an observational longitudinal study including patients visiting 5 memory clinics in 3 Nordic countries. The maximum follow-up time was 7 years, and mean follow-up time was 62.5 (SD 17.6) months.

\section{Participants}

From a previous qEEG validity study in 6 Nordic clinics, consisting of 377 patients and $146 \mathrm{HC}$, we included all patients with a diagnosis of SCD and MCI from 5 of those clinics [35]. Patients with a primary diagnosis of depression of moderate or severe degree, bipolar disorder, or a psychotic disorder were excluded. In addition, we at random included a group of $\mathrm{HC}$ from the previous study. This resulted in 47 patients with SCD, 99 with MCI, and 67 $\mathrm{HC}$, a total of 213 participants. The participating sites were the university memory clinics in Oslo and Bergen (Norway), Copenhagen and Roskilde (Denmark), and Reykjavik (Iceland). For 13 patients, we could not retrieve valid follow-up data because of death or because the participants declined reexamination. Among these, the diagnosis was SCD in 2 and MCI in 11 patients. They did not differ regarding age, gender, education level, marital status, or cognition compared to the included SCD and MCI patients. Thus, the analyses were made with data from 200 participants, 45 with SCD, 88 with MCI, and $67 \mathrm{HC}$.

\section{Assessments}

A comprehensive clinical assessment was conducted at baseline and follow-up, except for those patients who had died during follow-up and for a few patients residing in nursing homes because of dementia of moderate or severe degree. For those, hospital and nursing home records were examined. The assessment was similar at the 5 academic centers and included a history from the 
patient and an informant, a physical examination, blood tests to screen for disorders associated with cognitive decline, neuropsychology to cover various cognitive domains, CT or MRI of the brain for evaluation of general atrophy and atrophy of the medial temporal lobes (MTA by Scheltens' scale [40]), and white matter changes according to the scale of Fazekas et al. [41]. Depression was assessed using a modified version of the MontgomeryAasberg Depression Rating Scale [42]. At baseline, each of the 10 items was assessed as present or absent, giving a minimum score of 0 and a maximum score of 10 . At follow-up, the full scoring procedure was used with a minimum score of 0 and a maximum of $60[42,43]$. If the basic assessment was not sufficient to make a diagnosis, lumbar puncture was done for examination of $\beta$-amyloid, total tau, and phosphorylated tau protein in cerebrospinal fluid, and/or FDG-PET or DaTScan were carried out.

The following cognitive tests were used in the analyses:

- The Mini Mental Status Examination, a test that has a minimum score of 0 and a maximum of 30 (a higher sore indicates better cognitive function) $[44,45]$;

- The clock drawing test (CDT), using the scoring instruction of Shulman [46], with a minimum score of 0 and a maximum score of 5 (a higher score indicates better function);

- The 10-word test of the Consortium to Establish Alzheimer Disease Registry (CERAD), using all 3 scorings: CERAD learning with a maximum score of 30 , CERAD recall with a maximum score of 10, and CERAD recognition with a maximum score of 20 [47] (a higher score on each indicates better cognition).

Details on the comprehensive assessment, which was similar in the clinics, can be found elsewhere [48].

\section{Diagnoses of the Clinical Sample}

Clinical diagnoses were made by at least 2 experienced physicians. MCI was diagnosed using the MCI criteria of Winblad et al. [10]. In patients referred to the memory clinic because of cognitive complaints who did not meet the Winblad criteria or criteria for dementia, the diagnosis was SCD, using criteria similar to those made by Jessen et al. [12]. At follow-up, AD was diagnosed according to the criteria established by McKhann et al. [49], whereas $\mathrm{VaD}$ was diagnosed by the NINDS-AIREN criteria, DLB according to the revised consensus criteria, and frontotemporal dementia by the Lund-Manchester criteria [13,49-52]. Dementias due to Parkinson disease were diagnosed according to the ICD-10 criteria [14]. Other dementia was used for unspecified dementia and dementia due to causes such as normal pressure hydrocephalus and dementia due to multiple sclerosis. All diagnoses were made independently of the qEEG results.

\section{Healthy Controls}

Details of HC recruitment are described in the previous paper [35]. They were interviewed, a history of previous and present disorders and use of drugs were recorded and tested with the MMSE, the CDT, and the CERAD-10 word test. Depression was assessed with the Montgomery-Aasberg Depression Rating Scale in the same way as in the patients. Persons with a result on a cognitive test below $1 \mathrm{SD}$ according to their age were excluded at baseline. At follow-up, they were examined comprehensively according to the standard set in the memory clinic if MCI or dementia was suspected.

\section{EEG Recordings}

EEGs were recorded using the NicoletOne EEG System from Natus ${ }^{\circledR}$. Subsequent analysis was done in the MATLAB environ- ment from MathWorks ${ }^{\circledR}$. The IS 10-20 system was used for electrode placement using 19 electrodes, and features are evaluated using the average montage. Two bipolar electrooculography channels and 1 electrocardiogram were applied to monitor artifacts. EEG was recorded for at least 3 min with the subjects at rest and eyes closed. The subjects were alerted if they became visibly drowsy, as drowsiness influences the recording.

\section{qEEG Data Analysis}

The specific section of each recording chosen for further processing was selected by a trained technician such that artifacts were minimal and the length of the section at least 150s. Prior to feature extraction, the chosen segment was preprocessed by applying a 8th-order Butterworth band-pass filter with the chosen band (0.1$70 \mathrm{~Hz}$ ) to eliminate potential low- and high-frequency disturbances from the signal. The features extracted from the EEG recording and used in the evaluation of the dementia index (DI) were retrieved according to the recommendations of the Pharmaco-EEG society [53]. The society recommends that the signal is segmented into 2 -s segments overlapping by $1 \mathrm{~s}$. The signal is then analyzed segment by segment, and the feature values are estimated by evaluating the expected value over all the segments. This can be achieved by various means. For instance, using the average value or an alternative robust measure. Using a robust measure minimizes the impact of outliers and hence reduces the influence of potential signal artifacts. We use the simplest robust estimate, that is, the median of the feature values. The features used are all related to the spectral properties of the recording. Discrete fast Fourier transform is applied to estimate the spectral properties of the signal [54]. The analysis relies on recordings from 19 electrodes. The electrodes used are named according to the international 1020 system [55]: Fp1, Fp2, F3, F4, C3, C4, P3, P4, O1, O2, F7, F8, T3, T4, T5, T6, Fz, Cz, and Pz. If the fast Fourier transform components for each of the electrodes, segments, and discrete frequencies considered are denoted by $\sigma_{c i j}$, where $c \in\{1,2, \ldots, 19\}$ indicates the channel, $i \in\{1, \ldots, N\}$ the segment of the $N$ segments considered, and $j \in\{1, \ldots, 90\}$ the discrete frequencies $(0.5,1, \ldots, 45 \mathrm{~Hz})$, the full spectral resolution covariance between channels $c$ and $k$ is then expressed by $x_{c k}^{i j}=\sigma_{c i j} \times \sigma_{k i j}^{*}$. These covariances constitute the base features used for analysis and evaluation of the classification index values. To determine the core features relied on, principal components (PCs) were determined based on the Mentis Cura database of EEG recordings. PC analysis was performed on data from dementia subjects in the database. This was done separately for each covariance. PCs were then ranked according to their individual discriminatory properties in separating the subjects in the database. The discriminatory properties were determined according to the area under curve (AUC) of the receiver-operating characteristic curve (ROC). We use the 2 best performing components from each of the covariances to extract the core features used for evaluation of the index. If $P_{c k \alpha j}$ denotes the 2 chosen PCs, $\alpha \in\{1,2\}$, for electrode pair $(c, k)$ at frequencies $j \in\{1, \ldots, 90\}$, the core features considered for analysis then become $C_{c k \alpha}=$ $E_{i}\left\{\sum_{j=1}^{90} x_{c k}^{i j} P_{c k \alpha j}\right\}$. Figure 1 illustrates typical examples of the PCs for 1 covariance, $P_{111 j}$ and $P_{112 j}$, which describe how the spectral bands are weighted for the analysis. The PCs can be related to the classical EEG power bands, $\delta(1-4 \mathrm{~Hz}), \theta(4-8 \mathrm{~Hz}), \alpha(8-13 \mathrm{~Hz})$, and $\beta$ $(13-30 \mathrm{~Hz})$, which are indicated in the figure. Then PC1 corresponds to the difference between the combined $\delta$ and $\theta$ power and the $\beta$ power, while PC2 is a weighted measure of the total power
40
Dement Geriatr Cogn Disord 2020;49:38-47 DOI: $10.1159 / 000508392$
Engedal et al. 
Fig. 1. Typical examples of the PCs for one covariance, $P_{111 j}$ and $P_{112 j}$ specifically. The classical EEG power bands $\delta, \theta, \alpha$, and $\beta$ are indicated. In terms of the classical bands, PC1 corresponds to the difference between the combined $\delta$ and $\theta$ power, and the $\beta$ power, while PC2 is a weighted measure of the total power.

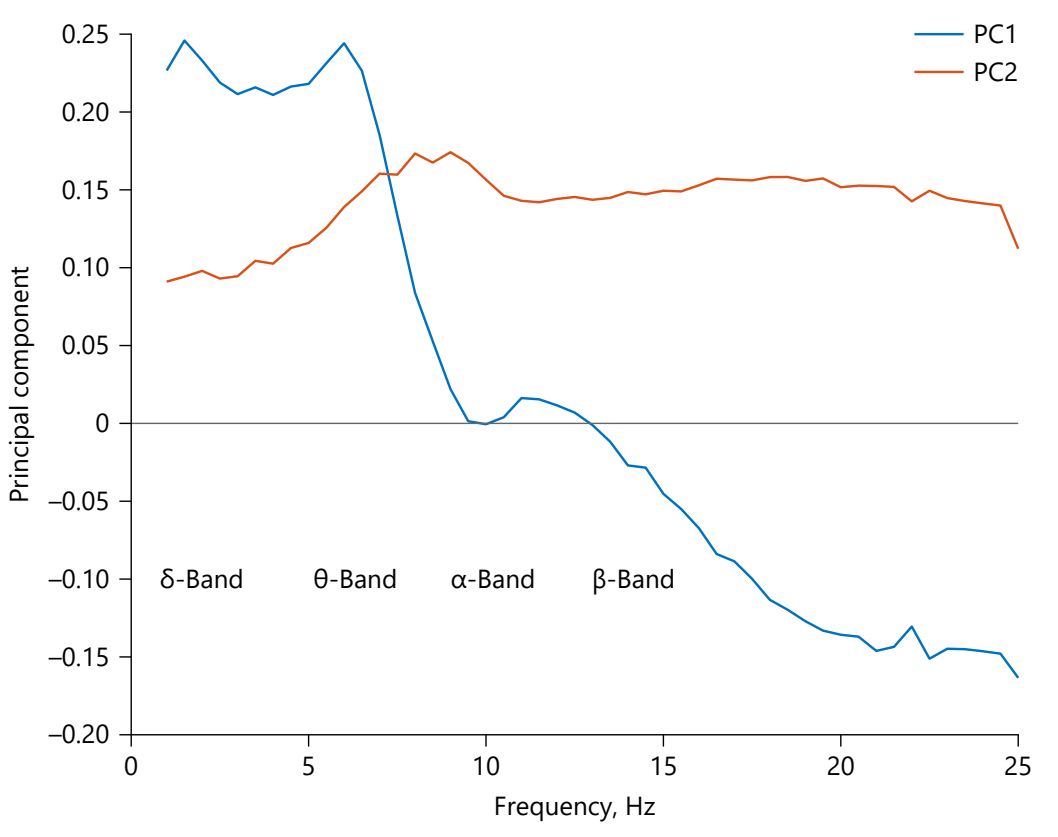

Table 1. Baseline characteristics of the 200 participants according to diagnosis at baseline

\begin{tabular}{lccccc}
\hline & All $(n=200)$ & HC $(n=67)$ & SCD $(n=45)$ & MCI $(n=88)$ & $p$ value \\
\hline Female & $105(53 \%)$ & $37(55 \%)$ & $24(53 \%)$ & $44(50 \%)$ & $0.800^{\mathrm{a}}$ \\
Married & $150(75 \%)$ & $49(73 \%)$ & $34(76 \%)$ & $67(76 \%)$ & $0.800^{\mathrm{a}}$ \\
Age, years & $68.7 \pm 8.2$ & $65.6 \pm 7.3$ & $68.0 \pm 8.0$ & $71.2 \pm 8.1$ & $<0.001^{\mathrm{b}}$ \\
Education, years & $12.3 \pm 3.9$ & $13.4 \pm 3.5$ & $12.9 \pm 3.8$ & $11.2 \pm 4.0$ & $0.001^{\mathrm{b}}$ \\
MMSE & $28.0 \pm 2.0$ & $29.0 \pm 1.1$ & $28.7 \pm 1.2$ & $27.0 \pm 2.2$ & $<0.001^{\mathrm{b}}$ \\
CDT & $4.6 \pm 0.8$ & $4.7 \pm 0.5$ & $4.8 \pm 0.6$ & $4.4 \pm 1.0$ & $0.003^{\mathrm{b}}$ \\
CERAD recall & $5.2 \pm 2.8$ & $7.3 \pm 1.8$ & $6.4 \pm 2.0$ & $3.4 \pm 2.3$ & $<0.001^{\mathrm{b}}$ \\
\hline
\end{tabular}

Numbers (\%) and means \pm SD are shown. ${ }^{a} \chi^{2}$ test, ${ }^{b}$ One-way ANOVA.

with slightly more emphasis on $\alpha$ and $\beta$ power. The index value for an individual recording is evaluated from these features by $I=$ $\sum_{c k \alpha} C_{c k \alpha} \beta_{c k \alpha}+\beta_{1}^{A} A+\beta_{2}^{A} A^{2}+\rho$, where $A$ is the age of the subject in years. The classification coefficients $\beta_{c k \alpha}, \beta_{i}^{A}$, and $\rho$ were determined using a combination of genetic algorithms to optimize the number of features used, and SVM (support vector machine), an SPR, was applied in the Mentis Cura database, which contains EEG data from people with various dementia diagnoses and HC. This was done separately for men and women, resulting in separate gender-dependent indices. It should be noted that the DI was developed to separate people with dementia from nondemented subjects and not to predict conversion from MCI to dementia.

\section{Statistics}

All data were analyzed using SPSS software. The DI data were close to normally distributed. To test for differences between groups, we used the independent $t$ test or one-way ANOVA $(>2$ groups) for continuous data. $\chi^{2}$ and Fisher exact test were used to compare groups for categorical data. The paired sample $t$ test was used to test for differences between baseline and follow-up cognitive test scores for converters and nonconverters, separately. Spearman's $\rho$ and linear regression analysis were used to examine variables associated with the DI. Logistic regression analysis was used to examine predictors of conversion to dementia. We did 2 separate analyses because the correlation between MMSE and CERAD recall was as high as 0.6 . As the 3 different scorings of the CERAD 10 word test correlated highly (CERAD learning vs. recall 0.79 ), we only used the CERAD recall score in the linear regression analyses as this is a measure of episodic memory. ROC analysis was applied to examine the discriminatory power of the DI to separate patients that converted to dementia from those who did not. We calculated the AUC, sensitivity, specificity, the likelihood ratio for a positive test, and the likelihood ratio for a negative test, and the cutoff of the DI that gave the best classification with a sensitivity of approximately $70 \%$. AUC should be significantly larger than 0.5 if the test (qEEG) is better then what is achieved by random. The likelihood for a 
Table 2. Participants $(n)$ from different diagnostic groups at baseline that converted to dementia, declined, remained stable, or recovered

\begin{tabular}{|c|c|c|c|c|c|}
\hline \multirow[t]{2}{*}{ Baseline } & \multicolumn{5}{|c|}{ Status at follow-up after a mean of 62.5 months } \\
\hline & normal & SCD & MCI & dementia & dementia diagnosis \\
\hline $\mathrm{HC}(n=67)$ & $62(92.5 \%)$ & $3(4.5 \%)$ & $1(1.5 \%)$ & $1(1.5 \%)$ & $\mathrm{AD}=1$ \\
\hline $\operatorname{SCD}(n=45)$ & $8(18.0 \%)$ & $24(53.0 \%)$ & $6(13.0 \%)$ & $7(16.0 \%)$ & $\mathrm{AD}=5, \mathrm{FTD}=1, \mathrm{OD}=1$ \\
\hline MCI $(n=88)$ & $2(2.0 \%)$ & $2(2.0 \%)$ & $22(25.0 \%)$ & $62(71.0 \%)$ & $\mathrm{AD}=47, \mathrm{FTD}=1, \mathrm{DLB} / \mathrm{PD}=3, \mathrm{VaD}=3$, mixed $\mathrm{AD} / \mathrm{VaD}=4, \mathrm{OD}=4$ \\
\hline
\end{tabular}

Numbers are shown. AD, Alzheimer disease; DLB, dementia with Lewy bodies; FTD; frontotemporal dementia; OD, other dementia; PD, Parkinson disease; $\mathrm{VaD}$, vascular dementia.

Table 3. Characteristics at baseline and follow-up of the 130 nonconverters compared to the 70 converters

\begin{tabular}{|c|c|c|c|c|c|c|}
\hline \multirow[t]{2}{*}{ Characteristics } & \multicolumn{3}{|l|}{ Baseline } & \multicolumn{3}{|l|}{ At follow-up } \\
\hline & nonconverter & converter & $p$ value & nonconverter & converter & $p$ value \\
\hline \multicolumn{7}{|l|}{ Cognition } \\
\hline MMSE score & $28.8 \pm 1.5$ & $26.8 \pm 2.1$ & $<0.001$ & $28.5 \pm 3.2$ & $21.8 \pm 4.8$ & $<0.001$ \\
\hline CDT score & $4.7 \pm 0.7$ & $4.3 \pm 1.0$ & $<0.001$ & $4.8 \pm 0.6$ & $3.2 \pm 1.4$ & $<0.001$ \\
\hline CERAD recall score & $6.6 \pm 2.3$ & $2.9 \pm 2.0$ & $<0.001$ & $6.7 \pm 2.1$ & $1.1 \pm 1.5$ & $<0.001$ \\
\hline \multicolumn{7}{|l|}{ Comorbidity } \\
\hline Hypertension & $52(40 \%)$ & $40(56 \%)$ & $<0.05$ & $47(39.5 \%)$ & $24(41 \%)$ & ns \\
\hline Any heart disease & $13(10 \%)$ & $16(22.5 \%)$ & $<0.05$ & $20(17 \%)$ & $15(25.5 \%)$ & ns \\
\hline Diabetes & $6(5 \%)$ & $6(8.5 \%)$ & ns & $6(5 \%)$ & $5(8.5 \%)$ & ns \\
\hline Depression & $18(14 \%)$ & $13(18 \%)$ & ns & $1(1 \%)$ & $17(29 \%)$ & 0.01 \\
\hline \multicolumn{7}{|l|}{ Use of drugs } \\
\hline Antipsychotics & $3(2 \%)$ & $0(0 \%)$ & & $0(0 \%)$ & $0(0 \%)$ & \\
\hline Antidepressants & $11(8.5 \%)$ & $16(22.5 \%)$ & $<0.01$ & $10(9 \%)$ & $8(17 \%)$ & \\
\hline Hypnotics/tranquilizer & $7(5 \%)$ & $6(8.5 \%)$ & & $6(5.5 \%)$ & $0(0 \%)$ & \\
\hline Antidementia drugs & $0(0 \%)$ & $2(3 \%)$ & & $1(1 \%)$ & $16(22.5 \%)$ & \\
\hline
\end{tabular}

Numbers (\%) and means \pm SD are shown. The independent $t$ test was used for continuous variables and the $\chi^{2}$ or Fisher exact test for categorical variables. Due to the small numbers of drugs, statistical analysis was not done. Intragroup comparisons (baseline vs. follow-up) were performed using paired sample $t$ test but are not shown. $p=0.8,0.9$, and 0.7 for MMSE, clock drawing test, and CERAD recall scores at baseline vs. follow-up, respectively, for nonconverters, and $p<0.001, p<0.001$, and $p<0.002$ for converters, respectively. ns, nonsignificant.

positive test $=$ probability of a positive result in a person with a disease/probability of a positive results in a person without the disease (true positive/false positive) $=$ sensitivity $/ 1-$ specificity. The likelihood for a negative test $=$ probability of a negative result in a person with a disease/probability of a negative results in a person without the disease (false negative/true negative) $=1-$ sensitivity/specificity. Accuracy was defined as the proportion of correctly classified participants (converters + nonconverters).

\section{Results}

Table 1 shows the baseline characteristics of the study participants. As can been seen, the MCI patients were significantly older, had less years of education, and had poorer cognition as measured by MMSE, CDT, and CERAD10 recall compared to the participants of the 2 other groups. It should be noted that 45 (45\%) of the MCI patients had a score between 28 and 30 on the MMSE, and $33(33 \%)$ had a score between 5 and 10 on CERAD recall.

\section{Conversion to Dementia}

At follow-up, 70 of 200 (35\%) had converted to dementia, 62 MCI patients (71\%), 7 with SCD (16\%), and 1 HC (1.5\%). The converters were significantly older (73.1 [SD 7.5] years), compared to nonconverters (66.1 [SD 7.5] years) and had less years of education (11.2 [SD 4.3] vs. 13.0 [SD 3.6] years). No difference was found between
Engedal et al. 
Table 4. Dementia index (mean \pm SD) at baseline among nonconverters and converters

\begin{tabular}{ll}
\hline & Dementia index \\
\hline All $(n=200)$ & $46.9 \pm 9.9$ \\
Nonconverters $(n=130)$ & $42.6 \pm 9.9$ \\
Converters & \\
$0-12$ months $(n=25)$ & $58.7 \pm 10.9$ \\
$13-24$ months $(n=16)$ & $51.4 \pm 11.9$ \\
$25-36$ months $(n=14)$ & $48.1 \pm 7.7$ \\
$37-48$ months $(n=6)$ & $47.8 \pm 6.8$ \\
$49-60$ months $(n=8)$ & $48.0 \pm 4.7$ \\
\hline
\end{tabular}

$p<0.001$, nonconverters vs. converters ( $t$ test). Comparison between the 5 groups of converters: ANOVA $p=0.0001$. For 1 converter we did not have exact information at the time to conversion.

gender as $51 \%$ converters and $53 \%$ nonconverters were women, and $77 \%$ converters were married versus $72 \%$ nonconverters. Details of the diagnostic status at followup can be found in Table 2, whereas Table 3 shows that the converters had poorer cognitive test scores than nonconverters both at baseline and follow-up and poorer cognition at follow-up compared to baseline, whereas that was not the case for the nonconverters. Time to conversion was $<1$ year for 25 patients and $<2$ years for 41 (Table 4).

\section{EEG DI Using SPR and Its Associates}

The baseline DI for the MCI patients was 49.8 (10.0), for the SCD 44.8 (9.7), and for the HC 41.9 (8.2). For the converters, the index at baseline was 52.1 (SD 10.4) versus 42.6 (SD 9.9) among the nonconverters (Fig. 2).

The highest correlation with the DI was found for age, $0.66(p<0.001)$, followed by the CERAD recall score at baseline, $-0.45(p<0.001)$, MMSE score at baseline, $-0.41(p<0.001)$, years of education, -0.27 $(p<0.001)$, and CDT score at baseline, $-0.14(p=0.012)$. A linear regression analysis, with the qEEG DI as the dependent variable and gender, age, education, MMSE score, and converters versus nonconverters as independent variables revealed that age $(p<0.001)$, MMSE score at baseline (0.02), and being a converter $(p=$ 0.004 ) were significantly associated with the DI. Educational level was not. Applying the CERAD recall score instead of MMSE at baseline showed that age $(p<$ $0.001)$, gender $(p=0.02)$, CERAD recall $(p=0.02)$, and being a converter $(p=0.04)$ were significantly associated with the DI.

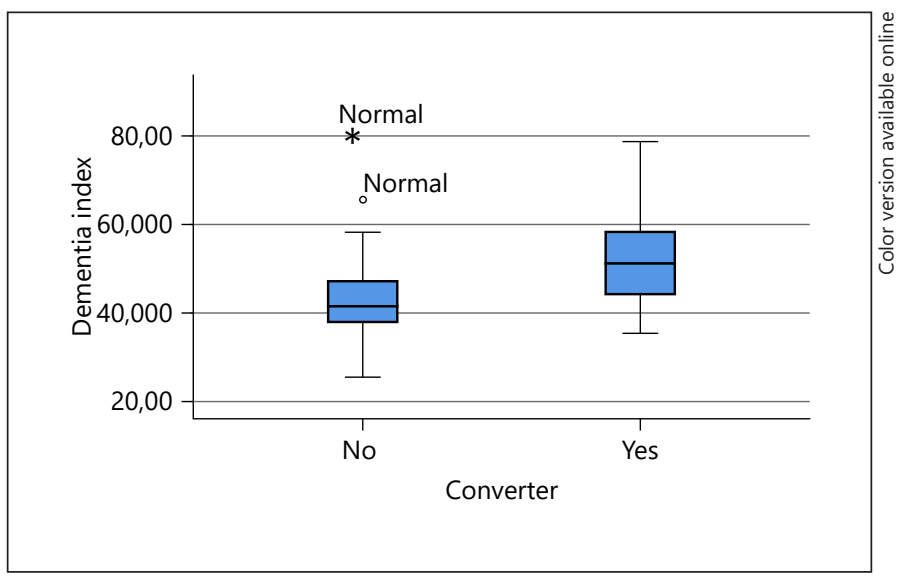

Fig. 2. Box plot of the dementia index at baseline for the group who converted (yes) and the group who did not convert to dementia (no) during follow-up.

Table 5 shows the qEEG DI for various age groups, educational levels, gender, MMSE score, and CERAD recall score for nonconverters and converters, separately. The index for those who developed AD was 52.5 (SD 4.6), for $\mathrm{VaD} 54.9$ (SD 3.1), for mixed $\mathrm{AD} / \mathrm{VaD} 52.5$ (SD 4.6), for DLB/Parkinson disease 46.5 (12.6), for frontotemporal dementia 41.6 (SD 8.7), and for the other dementia group 50.5 (7.7). The DI was highest for those who converted within the first or second year after the baseline examination (Table 4). For patients who converted to AD within 2 years, the DI was 56.5, whereas it was 46.6 for those who converted after 2 years.

\section{The Power of the DI to Separate Converters from \\ Nonconverters}

The results of the ROC analyses revealed that the DI had moderate power to separate the converters from nonconverters regardless if all participants were included, those who converted to dementia within the 2 first years, or those who developed $\mathrm{AD}$ (Table 6). Logistic regression analysis using converters versus nonconverters as dependent variable and DI, MMSE score, age, gender, and education showed that both the MMSE score (OR 0.62, 95\% CI $0.49-0.79, p<0.001$ ) and the DI (OR 1.1, 95\% CI $1.002-1.13, p=0.005$ ) were significant predictors of conversion to dementia with a correct classification rate of $78 \%$. Replacing the MMSE score with the CERAD recall score at baseline, the CERAD recall (OR 0.56, 95\% CI $0.46-0.68, p<0.001$ ), and the DI (OR $1.06,95 \%$ CI $1.02-$ $1.12, p=0.04)$ significantly predicted dementia with a correct classification rate of $84 \%$. 
Table 5. Dementia index (mean \pm SD) associated with gender, age group, educational level, MMSE, and CERAD recall score at baseline

\begin{tabular}{|c|c|c|c|c|c|c|}
\hline & All & $p$ value & Nonconverters & $p$ value & Converters & $p$ value \\
\hline \multicolumn{7}{|l|}{ Gender } \\
\hline Female & $50.7 \pm 9.7$ & \multirow[t]{2}{*}{0.8} & $42.8 \pm 9.1$ & \multirow[t]{2}{*}{0.8} & $55.0 \pm 10.4$ & \multirow[t]{2}{*}{0.02} \\
\hline Male & $48.5 \pm 14.7$ & & $42.4 \pm 5.8$ & & $49.2 \pm 9.9$ & \\
\hline \multicolumn{7}{|l|}{ Age groups } \\
\hline$<65$ years & $39.7 \pm 7.7$ & \multirow[t]{2}{*}{$<0.001$} & $39.3 \pm 8.2$ & \multirow[t]{2}{*}{$<0.001$} & $42.3 \pm 4.7$ & \multirow[t]{2}{*}{0.004} \\
\hline$>65$ years & $48.8 \pm 9.6$ & & $44.7 \pm 6.6$ & & $53.5 \pm 10.4$ & \\
\hline \multicolumn{7}{|l|}{ Education } \\
\hline$<10$ years & $50.7 \pm 12.5$ & \multirow[t]{2}{*}{$<0.001$} & $46.2 \pm 12.1$ & \multirow[t]{2}{*}{0.03} & $55.2 \pm 12.2$ & \multirow[t]{2}{*}{0.09} \\
\hline$>10$ years & $44.9 \pm 8.8$ & & $41.9 \pm 6.6$ & & $50.7 \pm 9.2$ & \\
\hline \multicolumn{7}{|l|}{ MMSE score } \\
\hline$<27$ & $54.2 \pm 9.8$ & \multirow[t]{2}{*}{$<0.001$} & $51.2 \pm 2.7$ & \multirow[t]{2}{*}{0.002} & $57.2 \pm 10.0$ & \multirow[t]{2}{*}{0.002} \\
\hline$>27$ & $44.4 \pm 9.0$ & & $42.1 \pm 7.7$ & & $49.2 \pm 9.6$ & \\
\hline \multicolumn{7}{|l|}{ CERAD recall } \\
\hline $0-4$ & 51.410 .1 & \multirow[t]{2}{*}{$<0.001$} & $49.3 \pm 11.9$ & \multirow[t]{2}{*}{0.006} & $52.2 \pm 10.6$ & \multirow[t]{2}{*}{0.8} \\
\hline $5-10$ & $43.0 \pm 8.4$ & & $41.8 \pm 7.6$ & & $50.0 \pm 9.3$ & \\
\hline
\end{tabular}

Table 6. ROC analysis of EEG dementia index (DI) as predictor of conversion to dementia

\begin{tabular}{|c|c|c|c|c|c|c|c|}
\hline & AUC $(95 \%$ CI $)$ & $\begin{array}{l}\text { SS, } \\
\%\end{array}$ & $\begin{array}{l}\mathrm{SP} \\
\%\end{array}$ & $\begin{array}{l}\text { Accuracy, } \\
\%\end{array}$ & $\mathrm{LR}+$ & LR- & $\begin{array}{l}\text { DI } \\
\text { cutoff }\end{array}$ \\
\hline Conversion including all participants & $0.78(0.70-0.85)$ & 71 & 68 & 69 & 2.2 & 0.43 & 44.8 \\
\hline Conversion to $\mathrm{AD}$ & $0.78(0.71-0.84)$ & 71 & 68 & 69 & 2.2 & 0.43 & 44.8 \\
\hline Conversion to $\mathrm{AD}, \mathrm{VaD}$, or mixed $\mathrm{AD} / \mathrm{VaD}$ & $0.79(0.73-0.86)$ & 73 & 70 & 71 & 2.4 & 0.39 & 45.3 \\
\hline Conversion from SCD to dementia & $0.83(0.64-1.0)$ & 67 & 64 & 65 & 1.9 & 0.52 & 46.6 \\
\hline
\end{tabular}

$\mathrm{AD}$, Alzheimer disease; AUC, area under the curve; LR+/LR-, positive/negative likelihood ratio; MCI, mild cognitive impairment; SCD, subjective cognitive decline; SP, specificity; SS, sensitivity; VaD, vascular dementia.

\section{Discussion}

To our knowledge, this is the first study on the discriminatory power of qEEG using the fully automated SPR method to predict conversion from SCD and MCI to dementia. It turned out that using the qEEG DI, the discriminatory power was of moderate degree, corresponding to a sensitivity of $71 \%$, specificity of $68 \%$, and accuracy of $69 \%$. It is difficult to compare our results with those of other EEG studies that have examined the power of EEG to predict conversion as the populations differ in size and cognition, the analytic methods differ, and ROC analyses have not always been done [26-30]. However, Poil et al. [26] could report a sensitivity of $88 \%$ and specificity of $87 \%$ applying several $\beta$-related frequencies (13$30 \mathrm{HZ}$ ), whereas Moretti [27] found the $\alpha 3 / \alpha 2$ frequency power ratio corresponded well with MRI findings indicating AD. Bonanni et al. [29] found that qEEG was perfect to predict DLB, with a sensitivity and specificity of $100 \%$. The fact that the DI was developed by cross-sectional analysis rather than by longitudinal follow-up could explain the modest discriminatory power.

The question arises whether a diagnostic test with a sensitivity of $71 \%$ and a specificity of $68 \%$ should be used in daily clinical practice. The DI should not be recommended as a prognostic instrument alone, neither in all participants nor in a subset of the participants (converting to $\mathrm{AD}$ or converting after 2 years). However, the EEG DI was associated with being a converter in the linear regression analyses, and in the logistic regression analysis the DI together with cognitive performance at baseline significantly predicted conversion to dementia, suggest-
44

Dement Geriatr Cogn Disord 2020;49:38-47 DOI: $10.1159 / 000508392$
Engedal et al. 
ing that both analyses indicate that the DI has an additional value in predicting conversion to dementia. In logistic regression analyses, we found that both MMSE and CERAD recall, when analyzed together with the DI, significantly predicted the conversion to dementia, whereas introducing medial temporal lobe atrophy measurements did not improve the results. The combination of CERAD recall and DI classified $84 \%$ of the participants correctly, whereas this rate was $78 \%$ for the combination of MMSE and DI. The better performance of the CERAD recall in combination with the DI than the MMSE is probably due to a ceiling effect using the MMSE, as $45 \%$ of the MCI patients scored above 27 on the MMSE. Another explanation could be that CERAD recall is more sensitive and primarily a test for episodic memory whereas MMSE measures cognition in more general terms. Episodic memory is the cognitive function that in most cases declines early in the development of $\mathrm{AD}$, the most frequent dementia disorder in the present study. Moreover, a systematic review made by the Cochrane group revealed that the MMSE is not a valid marker of conversion from MCI to dementia [56]. But, the results from the logistic regression analyses should be interpreted with caution as the cognitive tests and MRI were used in the diagnostic assessment.

It is difficult to compare our results with studies examining MRI, FDG and amyloid PET, and CSF biomarkers as predictors of conversion to dementia, because different populations and different statistical methods are used. Recently, one study using a machine learning program to evaluate structural brain changes using MRI as predictors of conversion reported very high AUC, and another study using voxel-based FDG-PET also reported very good results $[19,21]$. Best results are though reported from studies using various biomarkers in combination $[24,25]$. However, to design a study to examine the discriminant power of a combination of biomarkers and a cognitive test is difficult due to a circularity problem. We cannot examine the discriminant power of a test or examination when the result of the same is used together with tests to make a valid etiological diagnosis of dementia.

In the present study, as much as $71 \%$ of the MCI patients converted to dementia through a mean observation period of 5 years, whereas $25 \%$ were stable, and $4 \%$ improved. Of the SCD patients, $16 \%$ converted, $53.6 \%$ were stable, and the remaining 31.4\% recovered (Table 2). Even with an observation time up to a maximum of 7 years, we consider that the rate of conversion was high for both the MCI and SDC group, and higher compared to most published studies [15]. The reason might be that the patients of the present study are referred to an academic clinic. In the Nordic countries, except for the larger cities in Denmark, primary care physicians usually assess, diagnose, and treat patients with typical signs of dementia, and refer only the more complicated patients to memory clinics [57-60]. It should also be noted that converters to dementia in this study had more often poor cognition and hypertension, and were older as compared to nonconverters (Table 3), which are risk factors for conversion, as reported in systematic reviews [15]. Further, converters had significantly higher consumption of antidepressants at baseline than nonconverters (Table 3), which indicates some predementia psychiatric symptoms.

\section{Limitations}

The study has the following limitations. Although at least 2 experienced physicians at each center made the clinical diagnoses, it is possible that some patients should have been given another diagnosis. We applied current clinical criteria for the diagnosis of SCD and MCI, but it is not always simple to separate patients with MCI from those with an early phase of a dementia disorder. In a clinical setting, as in the present study, patients with an unsecure diagnosis are followed up. All patients had either a CT or MRI examination, but not all had CSF and PET examination, which could have improved the diagnostic accuracy. However, by inspecting the MMSE, CDT, and CERAD recall scores (Table 1), most included SCD and MCI patients had good cognitive function at baseline, which renders a diagnosis of dementia at baseline unlikely. In addition, the converters had a significant decline in all 3 cognitive tests at follow-up, whereas the nonconverters did not decline at follow-up (Table 3). These findings strengthen our belief that the clinical diagnoses were correct both at baseline and at follow-up. Further strengths of the study are the fairly large number of patients and $\mathrm{HC}$ and that the patients were included in an unselected manner, recruited from everyday clinical practice.

\section{Conclusion}

qEEG using the automated SPR method can be helpful in identifying patients with SCD and MCI that have a high risk of converting to dementia over a 5 -year period. However, as the discriminant power of the method is of moderate degree, it should be added to other routine diagnostic methods like cognitive tests and other biomarkers. 
Published in Celebration of the 30th Anniversary of the inception of Dementia and Geriatric Cognitive Disorders 1990-2020.

\section{Statement of Ethics}

All participants, or a family caregiver, were given oral and written information, and they consented to participation in writing in compliance with the World Medical Association Declaration of Helsinki. The study was approved by the Ethics Committees of the 3 countries, respectively.

\section{Disclosure Statement}

None of the authors have conflicts of interest.

\section{Funding Sources}

The Kavli Charitable Trust in Bergen, Norway, provided funding for the study.

\section{Author Contributions}

K.E. contributed to the study design, did the analyses, evaluated the analyses, wrote the preliminary and last draft of the manuscript, and approved it. M.L.B., P.H., B.B.A., M.N., A.-R.Ø., L.-O.W., and J.S. took part in the study design, evaluated the analyses, gave input to the preliminary and last draft of the manuscript, and approved it. N.W.D. and T.E.G. gave input to the preliminary and last draft of the manuscript, and approved it.

\section{References}

1 World Health Organization. Dementia fact sheet 2017. Available from: http://www.who. $\mathrm{int} /$ mediacentre/factsheets/fs362/en/.

2 Alzheimer's Disease International. World Alzheimer Report 2015. Available from: http://www.alz.co.uk/research/WorldAlzheimerReport2015.pdf.

3 Prince M, Bryce R, Albanese E, Wimo A, Ribeiro W, Ferri CP. The global prevalence of dementia: a systematic review and metaanalysis. Alzheimers Dement. 2013 Jan;9(1):63-75.e2.

4 Sperling RA, Aisen PS, Beckett LA, Bennett DA, Craft S, Fagan AM, et al. Toward defining the preclinical stages of Alzheimer's disease: recommendations from the National Institute on $\mathrm{Ag}$ ing-Alzheimer's Association workgroups on diagnostic guidelines for Alzheimer's disease. Alzheimers Dement. 2011 May;7(3):280-92.

5 Visser PJ, Kester A, Jolles J, Verhey F. Tenyear risk of dementia in subjects with mild cognitive impairment. Neurology. 2006 Oct; 67(7):1201-7.

6 Mauri M, Sinforiani E, Zucchella C, Cuzzoni MG, Bono G. Progression to dementia in a population with amnestic mild cognitive impairment: clinical variables associated with conversion. Funct Neurol. 2012 Jan-Mar;27(1):49-54.

7 Pandya SY, Lacritz LH, Weiner MF, Deschner M, Woon FL. Predictors of Reversion from Mild Cognitive Impairment to Normal Cognition. Dement Geriatr Cogn Disord. 2017; 43(3-4):204-14.

8 Gao Q, Gwee X, Feng L, Nyunt MS, Feng L, Collinson SL, et al. Mild Cognitive Impairment Reversion and Progression: Rates and Predictors in Community-Living Older Persons in the Singapore Longitudinal Ageing Studies Cohort. Dement Geriatr Cogn Disord Extra. 2018 Jun;8(2):226-37.

9 Petersen RC. Clinical practice. Mild cognitive impairment. N Engl J Med. 2011 Jun;364(23): 2227-34.
10 Winblad B, Palmer K, Kivipelto M, Jelic V, Fratiglioni L, Wahlund LO, et al. Mild cognitive impairment-beyond controversies, towards a consensus: report of the International Working Group on Mild Cognitive Impairment. J Intern Med. 2004 Sep;256(3):240-6.

11 Albert MS, DeKosky ST, Dickson D, Dubois B, Feldman HH, Fox NC, et al. The diagnosis of mild cognitive impairment due to Alzheimer's disease: recommendations from the National Institute on Aging-Alzheimer's Association workgroups on diagnostic guidelines for Alzheimer's disease. Alzheimers Dement. 2011 May;7(3):270-9.

12 Jessen F, Amariglio RE, van Boxtel M, Breteler M, Ceccaldi M, Chételat G, et al.; Subjective Cognitive Decline Initiative (SCD-I) Working Group. A conceptual framework for research on subjective cognitive decline in preclinical Alzheimer's disease. Alzheimers Dement. 2014 Nov; 10(6):844-52.

13 American Psychiatric Association. Diagnostic and statistical manual of mental disorders. 5th ed. Washington (DC): American Psychiatric Publishing; 2013.

14 World Health Organization. The ICD-10 Classification of Mental and Behavioural Disorders: Clinical descriptions and diagnostic guidelines. Geneva: World Health Organization; 1992.

15 Roberts R, Knopman DS. Classification and epidemiology of MCI. Clin Geriatr Med. 2013 Nov;29(4):753-72.

16 Pagani M, De Carli F, Morbelli S, Öberg J, Chincarini A, Frisoni GB, et al. Volume of interest-based $[18 \mathrm{~F}]$ fluorodeoxyglucose PET discriminates MCI converting to Alzheimer's disease from healthy controls. A European Alzheimer's Disease Consortium (EADC) study. Neuroimage Clin. 2014 Nov; 7:34-42.

17 Caroli A, Prestia A, Wade S, Chen K, Ayutyanont N, Landau SM, et al. Alzheimer Disease
Neuroimaging Initiative. Alzheimer Disease Biomarkers as Outcome Measures for Clinical Trials in MCI. Alzheimer Dis Assoc Disord. 2015 Apr-Jun;29(2):101-9.

18 La Rosa F, Saresella M, Baglio F, Piancone F, Marventano I, Calabrese E, et al. Immune and Imaging Correlates of Mild Cognitive Impairment Conversion to Alzheimer's Disease. Sci Rep. 2017 Dec;7(1):16760.

19 Moradi E, Pepe A, Gaser C, Huttunen H, Tohka J; Alzheimer's Disease Neuroimaging Initiative. Machine learning framework for early MRI-based Alzheimer's conversion prediction in MCI subjects. Neuroimage. 2015 Jan;104:398-412.

$20 \mathrm{Wu} \mathrm{A}$, Sharrett AR, Gottesman RF, Power MC, Mosley TH Jr, Jack CR Jr, et al. Association of Brain Magnetic Resonance Imaging Signs With Cognitive Outcomes in Persons With Nonimpaired Cognition and Mild Cognitive Impairment JAMA Netw Open. 2019 May;2(5):e193359.

21 Sörensen A, Blazhenets G, Rücker G, Schiller F, Meyer PT, Frings L; Alzheimer's Disease Neuroimaging Initiative. Prognosis of conversion of mild cognitive impairment to $\mathrm{Alz}$ heimer's dementia by voxel-wise Cox regression based on FDG PET data. Neuroimage Clin. 2019;21:101637.

22 Blennow K, Shaw LM, Stomrud E, Mattsson $\mathrm{N}$, Toledo JB, Buck K, et al. Predicting clinical decline and conversion to Alzheimer's disease or dementia using novel Elecsys $A \beta(1-42)$, pTau and tTau CSF immunoassays. Sci Rep. 2019 Dec;9(1):19024.

23 Smailagic N, Lafortune L, Kelly S, Hyde C, Brayne C. 18F-FDG PET for Prediction of Conversion to Alzheimer's Disease Dementia in People with Mild Cognitive Impairment: An Updated Systematic Review of Test Accuracy. J Alzheimers Dis. 2018;64(4): 1175-94. 
24 Zhou H, Jiang J, Lu J, Wang M, Zhang H, Zuo C; Alzheimer's Disease Neuroimaging Initiative. Dual-Model Radiomic Biomarkers Predict Development of Mild Cognitive Impairment Progression to Alzheimer's Disease. Front Neurosci. 2019 Jan;12:1045.

25 Ottoy J, Niemantsverdriet E, Verhaeghe J, De Roeck E, Struyfs H, Somers C, et al. Association of short-term cognitive decline and MCIto-AD dementia conversion with CSF, MRI, amyloid- and 18F-FDG-PET imaging. Neuroimage Clin. 2019;22:101771.

26 Poil SS, de Haan W, van der Flier WM, Mansvelder HD, Scheltens P, Linkenkaer-Hansen K. Integrative EEG biomarkers predict progression to Alzheimer's disease at the MCI stage. Front Aging Neurosci. 2013 Oct;5:58.

27 Moretti DV. Conversion of mild cognitive impairment patients in Alzheimer's disease: prognostic value of Alpha3/Alpha2 electroencephalographic rhythms power ratio. Alzheimers Res Ther. 2015 Dec;7(1):80.

28 Moretti DV. Association of EEG, MRI, and regional blood flow biomarkers is predictive of prodromal Alzheimer's disease. Neuropsychiatr Dis Treat. 2015 Oct;11:2779-91.

29 Bonanni L, Perfetti B, Bifolchetti S, Taylor JP, Franciotti R, Parnetti L, et al. Quantitative electroencephalogram utility in predicting conversion of mild cognitive impairment to dementia with Lewy bodies. Neurobiol Aging. 2015 Jan;36(1):434-45.

30 Cassani R, Estarellas M, San-Martin R, Fraga FJ, Falk TH. Systematic Review on RestingState EEG for Alzheimer's Disease Diagnosis and Progression Assessment. Dis Markers. 2018 Oct;2018:5174815.

31 Gudmundsson S, Runarsson TP, Sigurdsson S, Eiriksdottir G, Johnsen K. Reliability of quantitative EEG features. Clin Neurophysiol. 2007 Oct;118(10):2162-71.

32 Snaedal J, Johannesson GH, Gudmundsson TE, Gudmundsson S, Pajdak TH, Johnsen K. The use of EEG in Alzheimer's disease, with and without scopolamine - a pilot study. Clin Neurophysiol. 2010 Jun;121(6):836-41.

33 Ommundsen N, Engedal K, Øksengård AR Validity of the quantitative EEG statistical pattern recognition method in diagnosing Alzheimer's disease. Dement Geriatr Cogn Disord. 2011;31(3):195-201.

34 Snaedal J, Johannesson GH, Gudmundsson TE, Blin NP, Emilsdottir AL, Einarsson B, et al. Diagnostic accuracy of statistical pattern recognition of electroencephalogram registration in evaluation of cognitive impairment and dementia. Dement Geriatr Cogn Disord. 2012;34(1):51-60.

35 Engedal K, Snaedal J, Hoegh P, Jelic V, Bo Andersen B, Naik M, et al. Quantitative EEG Applying the Statistical Recognition Pattern Method: A Useful Tool in Dementia Diagnostic Workup. Dement Geriatr Cogn Disord. 2015;40(1-2):1-12.
36 Ferreira D, Jelic V, Cavallin L, Oeksengaard AR, Snaedal J, Høgh P, et al. Electroencephalography Is a Good Complement to Currently Established Dementia Biomarkers. Dement Geriatr Cogn Disord. 2016;42(1-2):80-92.

37 Schjønning Nielsen M, Simonsen AH, Siersma V, Engedal K, Jelic V, Andersen BB, et al. Quantitative Electroencephalography Analyzed by Statistical Pattern Recognition as a Diagnostic and Prognostic Tool in Mild Cognitive Impairment: Results from a Nordic Multicenter Cohort Study. Dement Geriatr Cogn Disord Extra. 2018 Nov;8(3):426-38.

38 Andersson M, Hansson O, Minthon L, Rosén I, Londos E. Electroencephalogram variability in dementia with lewy bodies, Alzheimer's disease and controls. Dement Geriatr Cogn Disord. 2008;26(3):284-90.

39 Bonanni L, Thomas A, Tiraboschi P, Perfetti B, Varanese S, Onofrj M. EEG comparisons in early Alzheimer's disease, dementia with Lewy bodies and Parkinson's disease with dementia patients with a 2-year follow-up. Brain. 2008 Mar;131(Pt 3):690-705.

40 Scheltens P, Leys D, Barkhof F, Huglo D, Weinstein HC, Vermersch P, et al. Atrophy of medial temporal lobes on MRI in "probable" Alzheimer's disease and normal ageing: diagnostic value and neuropsychological correlates. J Neurol Neurosurg Psychiatry. 1992 Oct;55(10):967-72.

41 Fazekas F, Chawluk JB, Alavi A, Hurtig HI, Zimmerman RA. MR signal abnormalities at $1.5 \mathrm{~T}$ in Alzheimer's dementia and normal aging. AJR Am J Roentgenol. 1987 Aug;149(2): 351-6.

42 Montgomery SA, Asberg M. A new depression scale designed to be sensitive to change. Br J Psychiatry. 1979 Apr;134(4):382-9.

43 Engedal K, Kvaal K, Korsnes M, Barca ML, Borza T, Selbaek G, et al. The validity of the Montgomery-Aasberg depression rating scale as a screening tool for depression in later life. J Affect Disord. 2012 Dec;141(2-3):227-32.

44 Folstein MF, Folstein SE, McHugh PR. "Minimental state". A practical method for grading the cognitive state of patients for the clinician. J Psychiatr Res. 1975 Nov;12(3):189-98.

45 Engedal K, Haugen P, Gilje K, Laake P. Efficacy of short mental tests in the detection of mental impairment in old age. Compr Gerontol A. 1988 Jun;2(2):87-93.

46 Shulman KI. Clock-drawing: is it the ideal cognitive screening test? Int J Geriatr Psychiatry. 2000 Jun;15(6):548-61.

47 Morris JC, Mohs RC, Rogers H, Fillenbaum G, Heyman A. Consortium to Establish a Registry for Alzheimer's Disease (CERAD) clini$\mathrm{cal}$ and neuropsychological assessment of Alzheimer's disease. Psychopharmacol Bull. 1988;24(4):641-52.

48 Brækhus A, Ulstein I, Wyller TB, Engedal K. The Memory Clinic-outpatient assessment when dementia is suspected. Tidsskr Nor Laegeforen. 2011 Nov;131(22):2254-7.
49 McKhann G, Drachman D, Folstein M, Katzman R, Price D, Stadlan EM. Clinical diagnosis of Alzheimer's disease: report of the NINCDS-ADRDA Work Group under the auspices of Department of Health and $\mathrm{Hu}$ man Services Task Force on Alzheimer's Disease. Neurology. 1984 Jul;34(7):939-44.

50 Román GC, Tatemichi TK, Erkinjuntti T, Cummings JL, Masdeu JC, Garcia JH, et al. Vascular dementia: diagnostic criteria for research studies. Report of the NINDS-AIREN International Workshop. Neurology. 1993 Feb;43(2):250-60.

51 McKeith IG, Dickson DW, Lowe J, Emre M, O'Brien JT, Feldman H, et al.; Consortium on DLB. Diagnosis and management of dementia with Lewy bodies: third report of the DLB Consortium. Neurology. 2005 Dec;65(12): 1863-72.

52 Neary D, Snowden JS, Gustafson L, Passant U, Stuss D, Black S, et al. Frontotemporal lobar degeneration: a consensus on clinical diagnostic criteria. Neurology. 1998 Dec;51(6): 1546-54.

53 Jobert M, Wilson FJ, Ruigt GS, Brunovsky M, Prichep LS, Drinkenburg WH; IPEG Pharmaco-EEG Guidelines Committee. Guidelines for the recording and evaluation of pharmaco-EEG data in man: the International Pharmaco-EEG Society (IPEG). Neuropsychobiology. 2012;66(4):201-20.

54 Cooley JW, Tukey JW. An algorithm for the machine calculation of complex Fourier series. Math Comput. 1965;19(2):297-301.

55 Chatrian GE, Lettich E, Nelson PL. Ten percent electrode system for topographic studies of spontaneous and evoked EEG activity. Am J EEG Technol. 1985;25(2):83-92.

56 Arevalo-Rodriguez I, Smailagic N, Roqué I Figuls M, Ciapponi A, Sanchez-Perez E, Giannakou A, et al. Mini-Mental State Examination (MMSE) for the detection of Alzheimer's disease and other dementias in people with mild cognitive impairment (MCI). Cochrane Database Syst Rev. 2015 Mar;(3):CD010783.

57 Olafsdóttir M, Skoog I, Marcusson J. Detection of dementia in primary care: the Linköping study. Dement Geriatr Cogn Disord. 2000 Jul-Aug;11(4):223-9.

58 Engedal K, Gausdal M, Gjøra L, Haugen PK. Assessment of dementia by a primary health care dementia team cooperating with the family doctor - the Norwegian model. Dement Geriatr Cogn Disord. 2012;34(5-6): 263-70.

59 Michelet M, Engedal K, Selbæk G, Lund A, Bjørkløf GH, Horndalsveen PO, et al. The Validity of the Norwegian Version of the Cognitive Function Instrument. Dement Geriatr Cogn Disord. 2018;46(3-4):217-28.

60 Westerlind B, Östgren CJ, Midlöv P, Marcusson J. Diagnostic failure of cognitive impairment in Nursing Home residents may lead to impaired medical care. Dement Geriatr Cogn Disord. 2019;47(4-6):209-18. 